\title{
Neoplasias malignas em cavidade oral associadas ao uso de narguilé: Revisão integrativa da literatura
}

\author{
Malignant neoplasms in oral cavity associated with the use of narguilé: \\ Integrative lliterature review
}

Luana Diniz ${ }^{1}$, Juliana Tomaz Sganzerla $2,3,4$

\section{RESUMO}

O tabaco é o principal fator de risco para o câncer bucal, sendo o narguilé um dispositivo utilizado para fumar tabaco, originário da Índia. De acordo com a Organização Mundial da Saúde, uma hora de sessão de narguilé equivale a fumar cem unidades de cigarros comuns. Acreditava-se que o narguilé causava menos danos à saúde do que o cigarro convencional, porém já existem dados científicos que destoam dessa crença. Julgavase, que as toxinas presentes na fumaça seriam "filtradas" ao passar pela água do dispositivo, no entanto, a água tem como função resfriar a fumaça, facilitando a inalação mais profunda, juntamente com as toxinas. Nessa perspectiva, avaliando o aumento do consumo do tabaco por meio do narguilé, essa revisão integrativa tem como objetivo pesquisar sobre neoplasias malignas em cavidade oral ocasionadas pelo uso do narguilé, e sua relação com o aumento do número de neoplasias malignas em cavidade oral.

Palavras-chave: Neoplasia Maligna; Câncer de Cabeça e Pescoço; Câncer Bucal; Narguilé; Shisha.

\section{ABSTRACT}

Tobacco is the main risk factor for oral cancer, with hookah/waterpipe being a device used to smoke tobacco, originally from India. According to the World Health Organization, an hour of hookah session is equivalent to smoking 100 units of ordinary cigarettes. It was believed that the hookah caused less damage to health than the conventional cigarette, but there are already scientific data that disagree with this belief. It was believed that the toxins present in the smoke would be 'filtered' when passing through the water of the device, however, the water has the function of cooling the smoke, facilitating deeper inhalation, together with the toxins. In this perspective, evaluating the increase in tobacco consumption through the hookah, this integrative review aims to research on malignant neoplasms in the oral cavity caused using the hookah, and its relationship with the increase in the number of malignant neoplasms in the oral cavity.

Keywords: neoplasms malignant, shisha, head and neck neoplasms, oral cancer e narghile
${ }^{1}$ Graduanda em Odontologia pela Universidade Luterana do Brasil, Campus Canoas - Rio Grande do Sul.

${ }^{2}$ Mestre, Docente do Curso de Odontologia da Universidade de Gurupi.

${ }^{3}$ Docente do Curso de Odontologia do Centro Universitário Luterano de Palmas (CEULP/ULBRA).

${ }^{4}$ Doutoranda do Curso de PósGraduação em Odontologia da Universidade Luterana do Brasil, Campus Canoas - Rio Grande do Sul.

E-mail:

julianasganzerla.js@gmail.com 


\section{INTRODUÇÄO}

O câncer oral é a sexta neoplasia maligna mais comum em todo o mundo. ${ }^{1}$ Há muito tempo é aceito que o consumo de tabaco, incluindo o fumo sem fumaça e o consumo excessivo de álcool, são os principais fatores etiológicos para o desenvolvimento do câncer oral. $^{2}$

Em relação à Odontologia, o tabaco está associado, entre outros, como fator de risco para o câncer bucal, ${ }^{3,4}$ à leucoplásia displásica ou não ${ }^{5}$, aumento na severidade e piora de prognóstico das doenças periodontais. ${ }^{6}$

Dentre as formas de uso do tabaco, o Narguilé é um dispositivo para fumar tabaco originário da Índia, no qual uma mistura de tabaco especial com essência é aquecida e a fumaça gerada passa por um filtro de água antes de ser aspirada pelo fumante, por meio de uma longa mangueira com piteira. ${ }^{7}$ Sua popularização nos últimos anos fez com que houvesse um aumento de consumo de tabaco, principalmente entre os jovens. A permissão de utilização em locais públicos e seu meio diferente de consumo podem ser um dos fatores de atratividade, principalmente ao público jovem. ${ }^{8}$

Por muito tempo acreditou-se que o narguilé causava menos danos à saúde do que o cigarro convencional, porém já existem dados científicos que destoam dessa crença. ${ }^{8}$ Julgava-se, de forma errônea, que as toxinas presentes na fumaça seriam "filtradas" ao passar pela água do dispositivo. ${ }^{9}$ No entanto, a água contida no narguilé tem como função resfriar a fumaça, facilitando a inalação mais profunda. Deste modo, o vapor penetra mais intensamente nos pulmões, carregando substâncias cancerígenas, metais pesados, inúmeras partículas tóxicas, além de altos níveis de nicotina. ${ }^{10}$

Diante disso e avaliando o aumento do consumo do tabaco por meio do narguilé, o objetivo dessa revisão integrativa da literatura é pesquisar sobre neoplasias malignas em cavidade oral ocasionadas pelo uso do narguilé e sua relação com o aumento do número de neoplasias malignas bucais em pacientes jovens.

\section{MATERIAIS E METODOS}

Trata-se de uma revisão integrativa da literatura abordando a ocorrência de neoplasias malignas em cavidade oral ocasionadas pelo uso do narguilé, realizada no período de janeiro a abril de 2021, utilizando uma busca na bases de dados US National Library of Medicine/National Institutes of Health (PubMed); Foram utilizados os descritores 
narghile, oral cancer, malignant neoplasms, indexados no vocabulário dos Descritores em Ciências da Saúde (DeCS).

Dois revisores foram treinados para seleção dos estudos a partir dos títulos e resumos, e de forma independente seguiram a estratégia de revisões sistemáticas. Após, os estudos selecionados a partir dos critérios de elegibilidade foram lidos na íntegra para análise e descrição dos resultados.

Foram utilizados como critérios de inclusão artigos originais com delineamento experimental (ensaios clínicos randomizados ou não), observacional (estudos transversais, de caso-controle e de coorte) e revisões sistemáticas, escritos em português, inglês e/ou espanhol. Foram excluídos estudos como em formas de cartas, artigos de opinião, comentários, publicações duplicadas, relatos e séries de casos, dissertações ou teses, estudos que não contemplem os critérios de inclusão e aqueles que não estavam disponíveis na íntegra.

\section{RESULTADOS E DISCUSSÄO}

Foram identificados 330 artigos potencialmente relevantes, desses 303 foram excluídos a partir do título e resumo (Figura 1). Dos 27 artigos selecionados para leitura na íntegra, 18 foram excluídos por não abordarem a ocorrência do narguilé com o câncer bucal. Com isso, restaram 9 artigos, que foram analisados e discutidos neste trabalho. $O$ quadro 1 apresenta a descrição dos artigos selecionados.

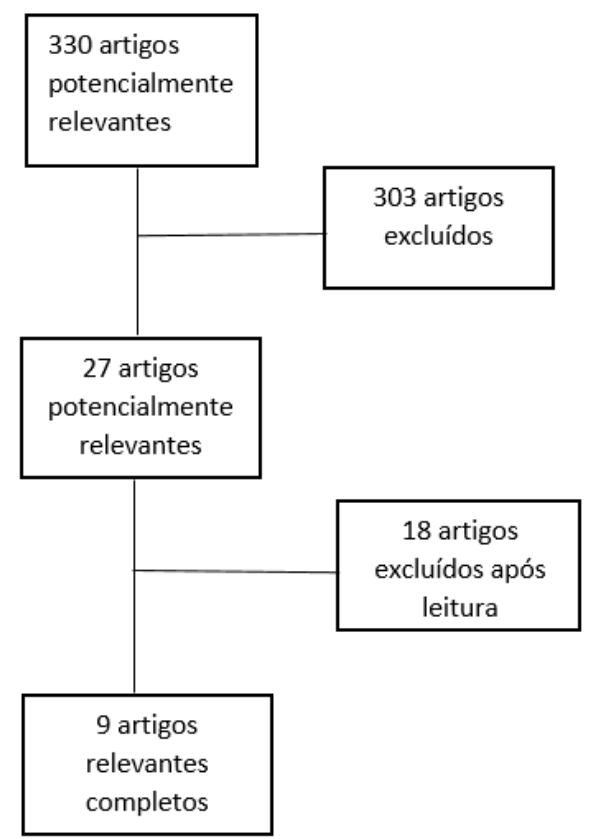

Figura 1. Fluxograma ae seıeçao e incıusao ae estuaos a parır aos crıterıos de elegibilidade. 
As evidências encontradas nos estudos analisados demonstraram que lesões orais potencialmente malignas e malignas (como leucoplasia e carcinoma de células escamosas oral, respectivamente) apresentam o uso do Narguilé como um fator de risco potencial para seu desenvolvimento, uma vez que os compostos aldeídos encontrados na fumaça de Narguilé são carcinogênicos e tóxicos à mucosa da cavidade bucal. ${ }^{12}$ Além disso, estimase que o uso de Narguilé e o consumo de álcool seja responsável por aproximadamente $75 \%$ dos cânceres da cavidade oral, em pacientes jovens. ${ }^{15}$

O uso do narguilé, que também é conhecido como Shisha, cria uma irritação celular que facilita o processo de envelhecimento das células da mucosa oral. Como resultado, as proteínas, que são sintetizadas dentro do núcleo, dividem-se lentamente, o que por sua vez gera atipia nuclear.

A hipótese para a associação entre uso de narguilé e câncer oral foi levantada em um estudo recente ${ }^{13}$ e demonstrou que pacientes com câncer bucal apresentavam frequência de uso de cigarro (66\%), narguilé (36\%) e álcool (17\%). A análise ajustada para sexo, tabagismo e consumo de álcool descobriu que os usuários de Narguilé eram significativamente mais jovens quando diagnosticados com câncer oral em comparação com os não usuários. Os resultados demonstram que o uso de narguilé é um fator de risco independente associado ao desenvolvimento de câncer bucal em idades mais jovens. ${ }^{13}$ Ainda, os resultados indicaram uma relação inversa entre a idade no diagnóstico do câncer oral e a frequência de fumar narguilé, ou seja, quanto mais frequentemente os pacientes com câncer oral fumavam narguilé, mais jovens eram ao serem diagnosticados com câncer. ${ }^{16}$

Quadro 1. Descrição dos estudos incluídos na revisão integrativa sobre neoplasias malignas bucais e uso de narguilé.

\begin{tabular}{|c|c|c|c|}
\hline Autor Principal & $\begin{array}{l}\text { País de } \\
\text { origem }\end{array}$ & $\begin{array}{c}\text { Ano de } \\
\text { publicação }\end{array}$ & Assunto abordado \\
\hline $\begin{array}{l}\text { Rebecca Pratiti } \\
11\end{array}$ & EUA & 2019 & $\begin{array}{l}\text { Epidemiologia e consequências adversas do uso de } \\
\text { narguilé }\end{array}$ \\
\hline Fawad Javed ${ }^{12}$ & EUA & 2017 & $\begin{array}{l}\text { Impacto toxicológico do fumo de narguilé na cavidade } \\
\text { oral e no sistema respiratório }\end{array}$ \\
\hline $\begin{array}{l}\text { Mehdi Khemiss } \\
13\end{array}$ & Tunísia & 2016 & Os efeitos do uso do narguilé na saúde bucal \\
\hline
\end{tabular}


DOI: 10.18606/2318-1419/amazonia.sci.health.v9n2p89-95 Revista Amazônia Science \& Health 2021, Vol. 9, № 2
DINIZ, L. SGANZERLA, JT.

Neoplasias Malignas em Cavidade Oral Associadas ao uso de Narguilé: Revisão Integrativa da Literatura

\begin{tabular}{|c|c|c|c|}
\hline $\begin{array}{l}\text { Shankargouda } \\
\text { Patil }{ }^{14}\end{array}$ & Itália & 2019 & $\begin{array}{l}\text { Alterações proteômicas em queratinócitos orais } \\
\text { cronicamente exposto ao Shisha (Narguilé) }\end{array}$ \\
\hline Khaled Zaid ${ }^{15}$ & Síria & 2018 & $\begin{array}{l}\text { Superexpressão de p53 na Mucosa Oral relacionada ao } \\
\text { hábito de fumar Shisha (Narguilé) na Síria e no Líbano }\end{array}$ \\
\hline $\begin{array}{l}\text { Suhail H. Al- } \\
\text { Amad }{ }^{16}\end{array}$ & $\begin{array}{l}\text { Emirados } \\
\text { Árabes } \\
\text { Unidos }\end{array}$ & 2014 & $\begin{array}{l}\text { Câncer oral em jovens jordanianos: possível associação } \\
\text { com a frequência de uso de narguilé }\end{array}$ \\
\hline H Ben Saad 17 & Tunisia & 2009 & Os efeitos do narguilé na saúde bucal \\
\hline $\begin{array}{l}\text { HatemW. Amer } \\
18\end{array}$ & Egito & 2018 & $\begin{array}{l}\text { Desenvolvimento de cancerização de campo na mucosa } \\
\text { oral clinicamente normal de fumantes de Shisha } \\
\text { (Narguilé) }\end{array}$ \\
\hline $\begin{array}{l}\text { Wasim Maziak } \\
19\end{array}$ & EUA & 2012 & Risco global emergente de câncer pelo uso de Narguilé \\
\hline
\end{tabular}

Ensaios laboratoriais in vitro foram realizados para investigar os efeitos protônicos sobre células expostas a Shisha e os resultados mostraram que os queratinócitos orais normais podem sofrer alterações celulares, através da desregulação da via do Interferon, regulação positiva das proteínas envolvidas no crescimento celular e regulação negativa dos processos imunológicos. Isso indica que as alterações celulares fenotípicas tendem a promover transformações oncogênicas pela exposição crônica ao extrato de Shisha. ${ }^{14}$

A mutação da proteína p53 (gene supressor tumoral, que desempenha um papel importante no controle do ciclo celular, no reparo do DNA e na indução da apoptose) foi associada ao fumo de Narguilé em pacientes com carcinoma espinocelular de boca e lesões potencialmente malignas, pois os fumantes de Shisha apresentaram um risco maior de mutação do p53 do que os não fumantes..$^{15}$ Outro fator importante encontrado foi a presença de displasia epitelial em $41 \%$ dos fumantes de cigarro e narguilé e em apenas $9 \%$ dos não fumantes. ${ }^{17,18}$, o que potencializa o risco de malignização dessas lesões.

Relatórios recentes têm demonstrado altos índices de câncer oral e orofaríngeo em países do Oriente Médio em comparação com outras partes do mundo, onde a exposição ao hábito do tabagismo é associada ao fumo de Narguilé. ${ }^{19}$ No Brasil, a popularização do Narguilé tem chamado a atenção da saúde pública para um problema que poderá tomar grandes proporções se não houver uma intervenção o mais breve possível, com a intenção de educar a população e atentá-la para o risco de desenvolvimento de câncer bucal, principalmente entre os jovens. 
DOI: 10.18606/2318-1419/amazonia.sci.health.v9n2p89-95 Revista Amazônia Science \& Health
DINIZ, L. SGANZERLA, JT.

Neoplasias Malignas em Cavidade Oral Associadas ao uso de Narguilé: Revisão Integrativa da Literatura

\section{CONSIDERAÇÕES FINAIS}

Ressalta-se que algumas limitações merecem ser citadas, como a não utilização de um instrumento para análise da qualidade dos artigos, assim como a inclusão de artigos com delineamentos distintos. Por conta disso, os resultados aqui apresentados devem ser analisados com cautela. Porém, ainda assim foi possível identificar que o narguilé pode ser considerado um agravante para o desenvolvimento de neoplasias malignas orais em adolescentes e adultos jovens, devido sua rápida disseminação e aceitação por parte do público, que erroneamente acredita ser menos prejudicial à saúde do que o cigarro convencional.

\section{REFERÊNCIAS}

1. S. Warnakulasuriya. Causes of oral cancer - an appraisal of controversies. College London Dental Institute. 2009

2. Brendan J. Perry. Andrew P. Zammit, BPharm; Andrew W. Lewandowski, Julia J. Bashford, Adrian S. Dragovic, Emily J. Perry, Reza Hayatbakhsh Sites of Origin of Oral Cavity Cancer in Nonsmokers vs Smokers: Possible Evidence of Dental Trauma Carcinogenesis and Its Importance Compared With Human Papillomavirus. JAMA Otolaryngology-Head \& Neck Surgery. 2015

3. Isabella Lima Arrais Ribeirol, Júlia Julliêta de Medeirosl, Larycia Vicente Rodriguesl, Ana Maria Gondim Valençal, Eufrásio de Andrade Lima Netol. Fatores associados ao câncer de lábio e cavidade oral. Universidade Federal da Paraíba - João Pessoa (PB) 2015

4. Instituto Nacional de Câncer (Brasil). Organização Pan-Americana da Saúde. Rio de Janeiro: INCA, 2011. 199p.

5. Joelma Sousa Lima, Décio dos Santos Pinto Jr, Suzana Orsini Machado de Sousa, Luciana Corrêa. Oral leukoplakia manifests differently in smokers and non-smokers. Oral Pathology Department, School of Dentistry, Univ of São Paulo, São Paulo, SP, Brazil. 2012

6. Drope J, Schluger N, Cahn Z, Drope J, Hamill S, Islami F, Liber A, Nargis N, Stoklosa M. The Tobacco Atlas. Atlanta: American Cancer Society and Vital Strategies.2018

7. W Maziak, K D Ward, R A Afifi Soweid, T Eissenberg. Tobacco smoking using a waterpipe: a re-emerging strain in a global epidemic. Virginia Commonwealth University and Syrian Center for Tobacco Studies. 2011.

8. Instituto Nacional de Câncer José Alencar Gomes da Silva. Narguilé: o que sabemos? / Instituto Nacional de Câncer José Alencar Gomes da Silva. - Rio de Janeiro: INCA, 2019. $100 \mathrm{p}$.

9. Alan Shihadeh, Jens Schubert, Joanne Klaiany, Marwan El Sabban, Andreas Luch, Najat A Saliba. Toxicant content, physical properties and biological activity of waterpipe 
DOI: 10.18606/2318-1419/amazonia.sci.health.v9n2p89-95 Revista Amazônia Science \& Health 2021, Vol. 9, № 2
DINIZ, L. SGANZERLA, JT.

Neoplasias Malignas em Cavidade Oral Associadas ao uso de Narguilé: Revisão Integrativa da Literatura

tobacco smoke and its tobacco-free alternatives. Department of Mechanical Engineering. 2015

10. Wasim Maziak, Ziyad Ben Taleb, Raed Bahelah,Farahnaz Islam, Rana Jaber,Rehab Auf, Ramzi G Salloum. The global epidemiology of waterpipe smoking. Department of Epidemiology, Robert Stempel College of Public Health and Social Work, Florida International University, Syrian Center for Tobacco Studies. 2014

11. Rebecca Pratiti,Debabrata Mukherjee. Epidemiology and Adverse Consequences of Hookah/Waterpipe Use: A Systematic Review. 2Texas Tech University Health Science Center,Department of Internal Medicine.2019

12. Fawad Javed. Toxicological impact of waterpipe smoking and flavorings in the oral cavity and respiratory system. Department of General Dentistry, Eastman Institute for Oral Health, University of Rochester, NY, USA. 2017

13. Mehdi Khemiss, Sonia Rouatbi, Latifa Berrezouga, Helmi Ben Saad. les effets de l'usage du narguilé sur l'etat bucco-dentaire Oral health effects associated with narghile use. Department of Dental Medicine, Fattouma BOURGUIBA Hospital, University of Monastir, Tunisia 2016.

14. Shankargouda Patil, Tejaswini Subbannayya,Sonali V. Mohan, Niraj Babu,Jayshree Advani, Gajanan Sathe,Pavithra Rajagopalan,Krishna Patel, Shilpa Bhandi, Hitendra Solanki, David Sidransky, Harsha Gowda, Aditi Chatterjee, and Marco Ferrari. Proteomic Changes in Oral Keratinocytes Chronically Exposed to Shisha (Water Pipe). A Journal of Integrative Biology Volume 23, Number 2, 2019

15. Khaled Zaid, Elie Azar-Maalouf, Charif Barakat, Mansour Chantiri. p53 Overexpression in Oral Mucosa in Relation to Shisha Smoking in Syria and Lebanon. Department of Oral Histology and Pathology, Faculty of Dentistry, Damascus University Damascus, Syria, 2018

16. Suhail H Al-Amad, Manal A Awad, Omar Nimri. Oral cancer in young Jordanians: potential association with frequency of narghile smoking. University of Sharjah, Sharjah, United Arab Emirates; Ministry of Health, Amman, Jordan 2014

17. H.Ben Saad. Le narguilé et ses effets sur la santé. Partie I : le narguilé, description générale et propriétésThe narghile and its effects on health. Part I: The narghile, general description and properties. Revue de Pneumologie Clinique, December 2009.

18. Hatem W. Amer, Hend M. Waguih, Dalia H. El-Rouby. Development of field cancerization in the clinically normal oral mucosa of shisha smokers. Oral and Maxillofacial Pathology Department, Faculty of Dentistry, Cairo University, Cairo, Egypt. 2018.

19. Wasim Maziak. The waterpipe: An emerging global risk for câncer. Department of Epidemiology, Robert Stempel College of Public Health and Social Work, Florida International University, Miami, USA. 2012. 